\title{
Effect of Oral Contraceptive Pill Pretreatment on Pregnancy Rates in Patients Stimulated with GnRH Antagonists and rFSH for ICSI
}

\author{
Dr. Soni Ashish Kumar ${ }^{1}$, Dr. Reddy Sanjeeva $\mathbf{N}^{2}$ \\ ${ }^{1}$ Assistant Professor Reproductive Medicine and Surgery Sriramachandra Institute of Higher Education \& Research \\ Chennai \\ ${ }^{2}$ HOD Reproductive Medicine and Surgery Sriramachandra Institute of Higher Education \& Research Chennai \\ Corresponding author: Dr. Ashish Kumar Soni (MCh); E2 SMART Sriramachandra Medical Centre Porur Chennai \\ (600116); ashish32257@gmail.com
}

Received 10 March 2020;

Accepted 29 March 2020;

Published 05 April 2020

\begin{abstract}
Background: After the recent introduction of GnRH antagonists in ovarian stimulation, OCP has been used for cycle scheduling purposes. Cycle programming has become more difficult with the use of GnRH antagonists, as stimulation initiation is dependent on the occurrence of menstruation. To overcome this limitation in the GnRH antagonist protocol, patients can be offered the use of pretreatment with oral contraceptive pills (OCP). Objective: To evaluate the effect of oral contraceptive pills (OCP) pretreatment on pregnancy rate in GnRH antagonist cycles. Design: Observational cohort study. Setting: Observational study performed at Sri Ramachandra institute of higher education \& research Chennai. Patients: Total 115 patients included in the study from January 2019 to December 2019.

All patients divided into two groups, oral contraceptives pretreated group (n-64) and oral contraceptives non treated group (n-51).

Results: All oral contraceptives pretreated patients required significantly higher dose of gonadotropins $(4745 \pm 1476$ versus $3659 \pm 1230 ; \mathrm{P}$ $<0.0005)$ and significantly longer days of stimulations $(12.2 \pm 1.2$ versus $10.5 \pm 0.8 ; \mathrm{P}<0.0005)$ in comparison to non-oral contraceptives treated group. There were no difference in total oocytes retrieved and fertilization rate. There were no other differences in cycle characteristics between groups. Implantation and pregnancy rates were not affected by OCP pretreatment. Conclusions: OCP pretreatment use for synchronization of follicles and cycle scheduling in GnRH-antagonist protocol, though it may be associated with longer stimulation and higher gonadotropin consumption but similar pregnancy rates.
\end{abstract}

Kevword: IVF, OCP, GnRH, Antagonist

\section{Introduction}

Gonadotropin-releasing hormone $(\mathrm{GnRH})$ antagonist protocols are characterized by shorter stimulation period and use of lower quantities of gonadotropins as compared with the long GnRHagonist protocol ${ }^{[1,3]}$. However, in a long GnRH-agonist protocol there is flexibility in the starting day of gonadotropin stimulation, which is lacking in the GnRH-antagonist protocol. To overcome this limitation in the GnRH antagonist protocol, patients can be offered the use of pretreatment with oral contraceptive pills (OCP) ${ }^{[4,7]}$. Moreover, a previous study has shown that OCP pretreatment before $\mathrm{GnRH}$ antagonist led to higher numbers of oocytes retrieved compared to the standard GnRH antagonist protocol ${ }^{[11]}$. On the other hand, longer stimulation periods and increased consumption of recombinant FSH (rFSH) were needed for stimulation ${ }^{[10,11]}$. The effect of this intervention on the probability of pregnancy has so far been examined only in a small randomized controlled trial (RCT).
The objective of the present study was to assess the effect of OCP pretreatment on pregnancy rates in patients stimulated with recombinant FSH (rFSH) and GnRH antagonist for IVF.

\section{Materials and methods}

Patients

Between Jan 2019 and Dec 2019, a GnRH-antagonist protocol was used in 115 patients. In 64 of these cycles, OCP pretreatment was administered for cycle scheduling. The use of pretreatment with $\mathrm{OCP}$ was for synchronisation of follicles before $\mathrm{COH}$.

\section{Ovarian stimulation protocols}

OCP pretreatment was administered for 21 days, starting on cycle days $2-3$. At the end of the OCP period prior to gonadotropin stimulation, vaginal ultrasound was performed to establish ovarian and uterine quiescence. Five days after OCP discontinuation, ovarian stimulation was commenced using $\mathrm{rFSH}$ at a starting dose 
of 225 to $300 \mathrm{IU} /$ day depending on age, AFC, AMH and D2 FSH $\&$ E2. The dose was adjusted after 5 days according to the patient's individual E2 response and follicular development. In the non-OCP protocol, gonadotropin stimulation was started on day 2 or 3 of the menstrual cycle, with a similar policy for the starting dose as in the OCP protocol. In both protocols, GnRH-antagonist was started when the leading follicle reached $\geq 14 \mathrm{~mm}$ in diameter, and was continued until the day of human chorionic gonadotropin (hCG) administration. Ovulation was triggered with $250 \mathrm{mcg}$ of $\mathrm{rHCG}$ when at least three follicles measuring $17 \mathrm{~mm}$ were detected by ultrasound scan. Oocyte collection, ICSI and luteal phase support were performed in the same manner in both protocols, in accordance with our hospital and laboratory standard of care and practice. Embryo transfer was deferred in patients who had an elevated progesterone on the day of hCG trigger $(>1.5 \mathrm{ng} / \mathrm{ml})$, or who had risk of OHSS or women who had an agonist trigger for final oocyte maturation or who had thin endometrium. Embryo transfer was done in the rest of patients who had no risk for embryo transfer failure. Upto 3 embryos were transferred on the second day or third day after oocyte retrieval. Serum $\beta$ hCG was measured on the 14th day of embryo transfer. Serum $\beta$ hCG values more than $25 \mathrm{mIU} / \mathrm{ml}$ was considered as positive test for pregnancy. Transvaginal sonography was done after 2 weeks of serum beta
HCG estimation to confirm site of pregnancy, number of sacs and cardiac activity.

\section{Statistical analysis}

The collected data were analysed with SPP statistics software 23.0 version. To describe about the data descriptive statistics frequency analysis, percentage analysis were used for categorical variables and the mean \& S.D were used continuous variables. To find significant difference between the bivariate samples in independent groups the unpaired t-test was used. To find the significance in categorical data Chi-Square test and Fishers exact test was used. In all above statistical tools the probability value 0.05 is considered as significant level.

\section{Results}

There were no differences within each age group between the pretreated OCP and non-OCP treated patients in demographic and baseline clinical parameters (Table 1\&2). The IVF cycle characteristics and laboratory data are presented in Table 3. The stimulation for the patients taking the oral contraceptive was on average of longer duration than for the Non-OCP group and pregnancy rates similar in both groups.

Table 1: Demographic characteristics between the groups

\begin{tabular}{|l|l|l|l|}
\hline DEMOGRAPHIC CHARACTERISTICS & NON OCP GROUP $(\mathbf{N}=\mathbf{5 1})$ & OCP GROUP B $(\mathbf{N}=\mathbf{6 4})$ & P value \\
\hline Age (years) & $29.49 \pm 3.5$ & $29.94 \pm 3.1$ & 0.47 \\
\hline Duration of infertility (years) & $7.8 \pm 4.4$ & $7.1 \pm 3.8$ & 0.66 \\
\hline BMI $(\mathrm{kg} / \mathrm{m} 2)$ & $26.55 \pm 5.3$ & $26.1 \pm 4.4$ & 0.40 \\
\hline
\end{tabular}

Table 2: Ovarian reserve between the groups

\begin{tabular}{|l|l|l|l|}
\hline OVARIAN RESERVE & NON OCP GROUP $(\mathbf{N}=\mathbf{5 1})$ & OCP GROUP $(\mathbf{N}=\mathbf{6 4})$ & P value \\
\hline D2 FSH $(\mathrm{mIU} / \mathrm{ml})$ & $6.6 \pm 2.2$ & $6.6 \pm 2.2$ & 0.95 \\
\hline D2 $\mathrm{LH}(\mathrm{mIU} / \mathrm{ml})$ & $4.9 \pm 2.9$ & $4.5 \pm 2.0$ & 0.34 \\
\hline D2 $\mathrm{E} 2(\mathrm{pg} / \mathrm{ml})$ & $48.07 \pm 17.5$ & $48.7 \pm 23.7$ & 0.94 \\
\hline AFC $(\mathrm{n})$ & $12.6 \pm 2.5$ & $12.6 \pm 3.4$ & 0.95 \\
\hline AMH $(\mathrm{ng} / \mathrm{ml})$ & $2.3 \pm 0.9$ & $2.4 \pm 1.01$ & 0.49 \\
\hline
\end{tabular}

Table 3: IVF cycle characteristics in OCP and non-OCP cycles

\begin{tabular}{|l|l|l|l|}
\hline PARAMETERS & NON OCP GROUP $(\mathbf{N}=\mathbf{5 1})$ & OCP GROUP $(\mathbf{N}=\mathbf{6 4})$ & P value \\
\hline Duration of stimulation (Days & $10.51 \pm 0.88$ & $12.2 \pm 1.26$ & 0.00 \\
\hline Total dose of Gn (IU) & $3659.80 \pm 1230.8$ & $4745.63 \pm 1476.01$ & 0.000 \\
\hline ET $(\mathrm{mm})$ & $10.04 \pm 1.8$ & $9.6 \pm 1.7$ & 0.21 \\
\hline E2 Trigger day (pg/ml) & $3732.55 \pm 1745.99$ & $2988.4 \pm 1434.13$ & 0.014 \\
\hline P4 on the day of trigger (ng/ml) & $1.13 \pm 0.5$ & $1.0 \pm 0.2$ & 0.14 \\
\hline NO.Oocytes & $16.02 \pm 7.1$ & $15.38 \pm 6.3$ & 0.59 \\
\hline Fertilization rate(\%) & $68 \%$ & $71 \%$ & 0.72 \\
\hline Implantation rate(\%) & $41 \%$ & $43 \%$ & 0.81 \\
\hline Pregnancy rate(\%) & $29 \%$ & $33 \%$ & 0.64 \\
\hline
\end{tabular}

There was no evidence of an 'OCP effect' on the number of oocytes retrieved, nor on the Fertilization, Implantation and Pregnancy rates.

\section{Discussion}

In this study we evaluated the effect of OCP pretreatment prior to GnRH-antagonist protocol for cycle scheduling in IVF treatment. We found that OCP pretreatment was associated with a longer length of stimulation and an increase in the total dose of gonadotropins needed for stimulation. The OCP pretreatment did not affect the magnitude of the ovarian response in terms of the number of oocytes retrieved. The implantation and pregnancy rates were not affected by OCP pretreatment. Endometrial thickness was not affected by OCP.
There is limited body of data in the literature on the use OCP pretreatment prior to $\mathrm{GnRH}$ antagonist protocol including three prospective randomized studies ${ }^{[10,11]}$. However, these studie include relatively small numbers of patients and cycles. Overall, our findings on the effect of OCP pretreatment prior to $\mathrm{GnRH}$ antagonist protocol on cycle characteristics, magnitude of ovarian response and pregnancy outcome are in accordance with these studies ${ }^{[10,11]}$. In all studies including this study, longer stimulation period and higher total dose of gonadotropins were needed in the OCP pretreatment cycles ${ }^{[8-10]}$. Similar to our results, in two studies ${ }^{[9,10]}$, OCP pretreatment had no effect on the final number of mature follicles whereas in one study ${ }^{[11]}$ the OCP pretreatment resulted in 
an increase in the number of mature follicles and in the number of oocytes retrieved. Finally, in our study as in previous studies, the implantation and pregnancy rates were not affected by the use of OCP pretreatment ${ }^{[9,10]}$.

\section{Conclusion}

OCP pretreatment for cycle scheduling in GnRH-antagonist protocol is a valid modality with comparable IVF outcome to the non-OCP protocol. The longer stimulation and higher total dose of FSH are the only drawbacks that we found in this modification. The weight of these drawbacks has to be measured against the gain in enabling cycle scheduling.

\section{References}

[1] Al-Inany H, Aboulghar M. GnRH antagonist in assisted reproduction: a Cochrane review. Hum Reprod $2002 ; 17: 874-85$

[2] Hohmann FP, Macklon NS, Fauser BC. A randomized comparison of two ovarian stimulation protocols with gonadotropin-releasing hormone $(\mathrm{GnRH})$ antagonist cotreatment for in vitro fertilization commencing recombinant follicle-stimulating hormone on cycle day 2 or 5 with the standard long GnRH agonist protocol. J Clin Endocinol Metab 2003;88:166-73

[3] Albano C, Felberbaum RE, Smitz J, Riethuller-Winzen $\mathrm{H}$, Engel J, Diedrich K, et al. Ovarian stimulation with HMG: results of a prospective randomized phase III European study comparing the luteinizing hormonereleasing hormone (LHRH)-antagonist cetrorelix and the LHRH-agonist buserelin. European Cetrorelix Study Group. Hum Reprod 2000;15:526-31.

[4] Obruca A, Fischl F, Huber JC. Scheduling OPU in GnRH antagonist cycles. J Reprod Fertil 2000;4:37.
[5] Cedrin-Durnerin I, Grange-Dujardin D, Laffy A, Parneix I, Massin N, Galey J, et al. Recombinant human LH supplementation during GnRH antagonist administration in IVF/ICSI cycles: a prospective randomized study. Hum Reprod 2004;19:1979-84

[6] Barmat LI, Chantilis SJ, Hurst BS, Dickey RP. A randomized prospective trial comparing gonadotropinreleasing hormone $(\mathrm{GnRH})$ antagonist/recombinant follicle-stimulating hormone ( $\mathrm{rFSH})$ versus $\mathrm{GnRH}-$ agonist/rFSH in women pretreated with oral contraceptives before in vitro fertilization. Fertil Steril 2005;83:321-30.

[7] Meldrum D, Scott R, Levy MJ, Alper M, Noyes N. A pilot study to assess oral contraceptive (OC) pretreatment in women undergoing controlled ovarian hyperstimulation $(\mathrm{COH})$ in ganirelix acetate cycles. Fertil Steril 2002;78(3 Suppl 1):S176 Abstract P-182.

[8] McCullagh P, Nelder JA. Generalised linear models. London: Chapman and Hall; 1989.

[9] Rombauts L, Healy D, Norman RJ, on the behalf of the Orgalutran Scheduling Study Group. A comparative randomized trial to assess the impact of oral contraceptive pretreatment on follicular growth and hormone profiles in $\mathrm{GnRH}$ antagonist-treated patients. Hum Reprod 2006;21:95-103.

[10] Kolibianakis EM, Papanikolaou EG, Camus M, Tournaye H, Van Steirteghem AC, Devroey P. Effect of oral contraceptive pill pretreatment on ongoing pregnancy rates in patients stimulated with $\mathrm{GnRH}$ antagonists and recombinant FSH for IVF. A randomized controlled trial. Hum Reprod 2006;21:352

[11] Huirne JA, Van Loenen ACD, Donnez J, Pirard C, Homburg R, Schats $\mathrm{R}$, et al. Effect of an oral contraceptive pill on follicular development in IVF/ICSI patients receiving a GnRH antagonist: a randomized study. Reprod Biomed Online 2006b;13(2):235-45 